\title{
VALORACIÓN NUTRICIONAL Y SENSORIAL DE PANQUECAS ELABORADAS A BASE DE HARINA DE TRIGO (TRITICUM AESTIVUM L.) Y ZANAHORIA (DAUCUS CAROTA L.)
}

\author{
NUTRITIONAL ASSESSMENT OF PANCAKE ELABORATED WITH WHEAT \\ FLOUR AND CARROT (DAUCUS CAROTA L.)
}

\author{
Luisa Gamboa ${ }^{1}$; Mario González ${ }^{1}$; Ernesto Hurtado ${ }^{2}$
}

\begin{abstract}
RESUMEN
Con el propósito de realizar la valoración nutricional de panquecas elaboradas a base de harina de trigo (HT) y zanahoria (Z) (Daucus carota $\mathrm{L}$.), se evaluaron cuatro tratamientos definidos por el nivel de sustitución de HT por trozos de $\mathrm{Z}$ en las panquecas (100\% HT-0\% Z; 75\% HT-25\% Z; 50\% HT-50\% Z; 25\% HT-75\% Z), utilizándose un diseño completamente aleatorizado. El análisis de varianza (ANAVA), aplicado a las variables dependientes estudiadas (humedad, proteína, grasa, fibra, cenizas, carbohidratos, $\beta$-caroteno y minerales), indicó diferencias significativas en ellas $(P<0,01)$, las cuales fueron analizadas por la prueba de Duncan, donde los porcentajes de fibra, cenizas (minerales), grasa, carbohidratos y contenido de $\beta$-caroteno aumentaron a medida que se incrementó el contenido de trozos de zanahoria en cada tratamiento y los de proteína y humedad disminuyeron con la sustitución parcial de la harina. Se determinó el contenido de $\beta$-caroteno a la mezcla de los ingredientes crudos y a las panquecas cocidas a $70^{\circ} \mathrm{C}$ y se detectó una reducción del $16 \%$ de este contenido por acción del cocimiento. Las variables cualitativas investigadas en la evaluación sensorial fueron analizadas a través de la prueba no paramétrica de KruskallWallis, resultando el tratamiento con 50\% HT y $50 \% \mathrm{Z}$ el de mayor aceptación. Los ensayos fueron realizados en el laboratorio de Tecnología de los Alimentos, de la Escuela de Zootecnia.
\end{abstract}

Palabras clave: Panquecas, zanahoria, $\beta$-caroteno, harina de trigo.

\begin{abstract}
In order to carry out the nutritious assessment of pancake elaborated with wheat flour and carrot, four defined treatments were evaluated by the level of replacement of wheat flour (WF) and carrot $(C)$ in pancakes. $(100 \%$ WF-0\% C; $75 \%$ WF-25\% C; $50 \% \mathrm{WF}-50 \% \mathrm{C} ; 25 \% \mathrm{WF}-75 \% \mathrm{C}$ ). A full-aleatory design was used. The varying analysis (ANOVA), applied to the dependent studied (humidity, protein, fat, fiber, ashen, carbohydrates, $\beta$-carotene and minerals), indicated significant differences in them $(P<0.01)$, which were analyzed by the test of Duncan, where the percentages of fiber, ashen (minerals), fat, carbohydrates and content of $\beta$-carotene, enlarged to the extent that the content of carrot was increased in each processing and those of protein and humidity decreased with the partial replacement of the flour. The content of $\beta$-carotene was determined to the mixture of the raw ingredients and to the cooked pancakes to $70^{\circ} \mathrm{C}$ and a reduction of the $16 \%$ of this content was detected of this contained by action of the thermal treatment. The qualitative variables investigated in the sensory evaluation were analyzed through the test non-parameter of Kruskall Wallis; resulting the processing with 50\% WF and 50\% C with higher acceptance. The tests were made in the laboratorie of Technology of Foods of the School of Zootecnia of the Universidad de Oriente, Monagas, Venezuela.
\end{abstract}

Key words: Pancakes, carrot, $\beta$-carotene, wheat flour.

1 Programa de Tecnología de los Alimentos. E-mail: luisa_beatrizg@ hotmail.com
2 Departamento de Biología y Sanidad Animal Escuela de Zootecnia. Universidad de Oriente. Monagas, Venezuela. E- mail:
ernestohurtado@ @antv.net

Fecha de Recepción: 27 Febrero 2006

Fecha de Aceptación: 05 Abril 2006 


\section{INTRODUCCIÓN}

La alimentación ha sido, a lo largo de la historia, una constante en las preocupaciones fundamentales del hombre. El desarrollo de las civilizaciones ha estado íntimamente ligado a su forma de alimentarse, incluso se dice que tal forma ha determinado el futuro o el destino de las mismas (Cheftel y Cheftel, 1992).

Actualmente se presenta una situación alimentaria-nutricional en la población tercermundista, con problemas de deficiencias de nutrientes, entre los que destacan la desnutrición por déficit de proteínas y energías, además la carencia de vitamina A y hierro. Estas deficiencias afectan principalmente a jóvenes y niños menores de cinco años (Méndez, 2001).

Muchos países en desarrollo y varias organizaciones internacionales (OAA/FAO, OMS, FDA, entre otros) se han preocupado en establecer estrategias para erradicar estas carencias nutricionales. Una de ellas consiste en el enriquecimiento y fortificación de alimentos de consumo masivo, para mejorar así la ingesta de vitamina $\mathrm{A}$, y otra incentivando el consumo de alimentos de origen vegetal, tal es el caso de la zanahoria, la cual es rica en $\beta$-caroteno, precursor de dicha vitamina (Méndez, 2001).

La panqueca de trigo es uno de los alimentos que se consume con frecuencia en el desayuno de países como México, Argentina, Estados Unidos, etc., gracias a su facilidad de preparación. Ésta puede mezclarse con otros tipos de alimentos (tanto de origen vegetal como de origen animal) para variar su sabor e incrementar su valor nutricional, entre ellos se destaca la zanahoria, la cual tiene múltiples posibilidades en la dieta. Maldonado y Pacheco (1998) la consideran como uno de los vegetales que presenta los mayores contenidos de fibra dietética natural y provitamina $\mathrm{A}$, de los cuales se han reportado valores muy altos con respecto a la gran mayoría de las hortalizas.

Aunado a esto, uno de los objetivos del estudio de los alimentos es desarrollar la capacidad de preparar y servir alimentos más apetitosos como lo señala Charley (2001). La tarea de mejorar el nivel de aceptación recae principalmente en los consumidores, lo cual contribuye en gran parte a fijar el estándar de sabor en el país; algunas de las formas para evaluar la calidad de un producto son: sensorialmente o subjetiva y otra utilizando medios químicos o instrumentos para cuantificar la composición nutricional.
Como contribución a la búsqueda de soluciones a problemas de carencias nutricionales en diversos alimentos, se plantea en esta investigación evaluar nutricional y sensorialmente panquecas elaboradas de harina de trigo sustituidas parcialmente con zanahoria.

\section{MATERIALES Y MÉTODOS}

\section{ELABORACIÓN DE LAS PANQUECAS}

Se elaboraron seis muestras de panquecas, $200 \mathrm{~g}$ aproximadamente por cada tratamiento, con la siguiente proporción porcentual en masa $(\mathrm{m} / \mathrm{m})$ de harina de trigo (HT) y trozos de zanahoria (Z), respectivamente: $100: 0 ; 75: 25 ; 50: 50 ; 25: 75 \%$ (tabla 1). A cada tratamiento se le adicionaron 50 $\mathrm{g}$ de huevo, $50 \mathrm{~g}$ de leche en polvo, $40 \mathrm{~g}$ de azúcar, $5 \mathrm{~g}$ de sal y $250 \mathrm{ml}$ de agua.

Los trozos de zanahorias previamente pesados según el tratamiento correspondiente y lavados se mezclaron con los ingredientes complementarios (huevo, leche, azúcar, sal y agua) en una licuadora a baja velocidad durante 2 minutos, luego se añadió harina de trigo comercial (todo uso) en las cantidades especificadas para cada tratamiento, se mezcló con un homogeneizador manual (batidor), durante 2 minutos. Posteriormente se sometieron a cocción en una cocina a $70^{\circ} \mathrm{C}$ durante 5 minutos, temperatura que fue controlada a través de un termómetro.

Tabla 1

Cantidades de harina de trigo (HT) y trozos de zanahoria $(Z)$ utilizadas como mezcla de ingredientes para la elaboración de panquecas de acuerdo a los tratamientos planteados

\begin{tabular}{|ccc|}
\hline Tratamientos & HT $(\mathbf{g})$ & $\mathbf{Z}(\mathbf{g})$ \\
\hline $100 \%$ HT; $0 \% \mathrm{Z}$ & 200 & 0 \\
\hline $75 \%$ HT; $25 \% \mathrm{Z}$ & 150 & 50 \\
\hline $50 \%$ HT; $50 \% \mathrm{Z}$ & 100 & 100 \\
$25 \%$ HT; $75 \% \mathrm{Z}$ & 50 & 150 \\
\hline
\end{tabular}

\section{EVALUACIÓN NUTRICIONAL}

- Determinación de la composición proximal de las panquecas: cada uno de los elementos fue cuantificado de acuerdo a lo establecido en 
las normas COVENIN (humedad 1.553-80, proteína $1.195-95$, grasa $1.785-81$, fibra cruda $1.789-81$, cenizas $1.783-81$ y carbohidratos por diferencia).

- Estipular el contenido de $\beta$-caroteno (Bushway, 1986): se realizó a través de la cromatografía líquida de alta resolución con detección espectrofotométrica, a $450 \mathrm{~nm}$. El método se basa en la extracción, en fase orgánica, de los carotenoides con tetrahidrofurano, con posterior separación de los carotenos mediante cromatografía líquida de alta resolución, en fase reversa, con elusión isocrática utilizando solución estándar de $\beta$-caroteno tipo IV.

- Determinación de minerales ( $\mathrm{Ca}, \mathrm{P}$ y Fe): se realizó según el método descrito por la Association of Analytical Chemists (AOAC, 1980). Método de extracción-digestión ácida.

\section{EVALUACIÓN SENSORIAL}

Se utilizó un panel semientrenado integrado por veinte (20) panelistas, para establecer cuál de los tratamientos tiene mayor aceptabilidad (sabor, olor, color y textura), evaluada a través de una escala hedónica (Mahecha, 1985)

\section{DISEÑO ESTADÍSTICO EXPERIMENTAL}

Se realizó un diseño completamente aleatorizado con cuatro tratamientos $(\mathrm{T} 1=100 \%$ HT Control, $\mathrm{T} 2=75 \%$ HT y $25 \% \mathrm{Z}, \mathrm{T} 3=50 \%$ HT y $50 \% \mathrm{Z}$, $\mathrm{T} 4=25 \%$ HT y $75 \% \mathrm{Z}$ ). A las variables paramétricas estudiadas (composición proximal, contenido de $\beta$-caroteno y contenido de minerales: $\mathrm{Ca}, \mathrm{P}, \mathrm{Fe})$ se les realizó un análisis de varianza (SAS, 1998). Se aplicó una prueba de promedios de rangos múltiples Duncan al 5\% para aquellos resultados donde las diferencias fueron significativas. Las variables cualitativas investigadas en la evaluación sensorial fueron analizadas a través de la prueba no paramétrica de Kruskall-Wallis (Chacín, 2000).

\section{RESULTADOS Y DISCUSIÓN}

\section{COMPOSICIÓN PROXIMAL}

Los resultados de la composición proximal de las panquecas $(P<0,01)$ elaboradas se muestran en la Tabla 2. Los valores de humedad decrecen a medida que disminuye el porcentaje de harina de trigo, lo cual podría atribuirse a la pérdida de agua durante el proceso de cocción por la baja capacidad de retención de agua de la zanahoria y al ser licuados sus trozos durante el mezclado libera fácilmente parte del agua (Belitz y Grosch (1997). Aunado a esto podríamos atribuir que las harinas son clasificadas de acuerdo al tipo de trigo, de aquí varía su capacidad de retención de agua; harinas elaboradas de trigo duro tienen mayor capacidad de agua y las elaboradas con trigo blando tienen menor capacidad de retención de agua (Charley, 2001).

Con respecto a la proteína cruda, en base húmeda, se observa una ligera disminución entre los diferentes tratamientos, a medida que disminuye el contenido de harina de trigo (todo uso), y

Tabla 2

Promedios de la composición proximal de las panquecas elaboradas bajo los tratamientos en estudio ${ }^{1 /}$

\begin{tabular}{|c|c|c|c|c|c|c|}
\hline Tratamientos & $\begin{array}{c}\text { Humedad } \\
\mathbf{\%}\end{array}$ & $\begin{array}{c}\text { Proteína } \\
\mathbf{\%}\end{array}$ & $\begin{array}{c}\text { Grasa } \\
\mathbf{\%}\end{array}$ & $\begin{array}{c}\text { Cenizas } \\
\mathbf{\%}\end{array}$ & $\begin{array}{c}\text { Fibra } \\
\mathbf{\%}\end{array}$ & $\begin{array}{c}\text { Carbohidratos } \\
\mathbf{\%}\end{array}$ \\
\hline $\begin{array}{c}100 \% \mathrm{HT} ; \\
0 \% \mathrm{Z}\end{array}$ & $51,97^{\mathrm{a}} \pm 0,27$ & $9,47^{\mathrm{a}} \pm 0,01$ & $1,51^{\mathrm{a}} \pm 0,04$ & $1,32^{\mathrm{a}} \pm 0,03$ & $0,43^{\mathrm{a}} \pm 0,01$ & $35,30^{\mathrm{a}} \pm 0,22$ \\
\hline $\begin{array}{c}75 \% \mathrm{HT} ; \\
25 \% \mathrm{Z}\end{array}$ & $47,54^{\mathrm{a}} \pm 0,79$ & $8,89^{\mathrm{a}} \pm 0,02$ & $2,21^{\mathrm{b}} \pm 0,05$ & $1,50^{\mathrm{a}} \pm 0,02$ & $0,77^{\mathrm{a}} \pm 0,01$ & $39,08^{\mathrm{a}} \pm 0,72$ \\
\hline $\begin{array}{c}50 \% \mathrm{HT} ; \\
50 \% \mathrm{Z}\end{array}$ & $43,15^{\mathrm{b}} \pm 0,79$ & $8,29^{\mathrm{a}} \pm 0,11$ & $2,65^{\mathrm{b}} \pm 0,00$ & $1,71^{\mathrm{a}} \pm 0,00$ & $2,42^{\mathrm{b}} \pm 0,08$ & $41,79^{\mathrm{b}} \pm 0,17$ \\
\hline $\begin{array}{c}25 \% \mathrm{HT} ; \\
75 \% \mathrm{Z}\end{array}$ & $36,38^{\mathrm{b}} \pm 0,06$ & $7,51^{\mathrm{b}} \pm 0,06$ & $3,17^{\mathrm{c}} \pm 0,00$ & $2,01^{\mathrm{a}} \pm 0,02$ & $2,77^{\mathrm{b}} \pm 0,03$ & $48,16^{\mathrm{b}} \pm 0,05$ \\
\hline
\end{tabular}

1/ Los valores son promedios de seis repeticiones en base húmeda \pm desviación estándar.

a, b, c Valores con diferentes letras en la misma columna difieren estadísticamente a una $P<0,05$. 
se aumenta el contenido de zanahoria $(1,0 \mathrm{~g} / 100$; INN, 2002), dichos valores no difieren estadísticamente con excepción del tratamiento $25 \% \mathrm{HT}$; $75 \%$ Z. Es importante mencionar el tipo de harina comercial utilizada, ya que ésta contiene mayor contenido de proteína que las harinas para elaborar pan y pasteles. El contenido de grasa, medido como extracto etéreo, se incrementa a medida que aumenta el contenido de zanahoria, los tratamientos $75 \%$ HT; $25 \% \mathrm{Z}$ y $50 \% \mathrm{HT} ; 50 \% \mathrm{Z}$ no difieren estadísticamente en comparación con los demás tratamientos. De todos los ingredientes utilizados, los que aportan la mayor cantidad de grasa son la leche y el huevo, pero sus cantidades son constantes en todos los tratamientos. El incremento observado podría atribuirse al aporte de carotenoides de la zanahoria, ya que estos pigmentos son solubles en grasas y se cuantifican junto a ella en el extracto etéreo (Belitz y Grosch, 1997).

En contenido de fibra cruda, en base húmeda, se observa un incremento desde 0,43 hasta $2,77 \%$, lo cual se debe al incremento en la proporción de zanahoria en los tratamientos. La fibra cruda es un indicativo del contenido de celulosa, hemicelulosa, pectinas y lignina. En relación con el contenido de cenizas, estos resultados indican la cantidad de materia inorgánica que pudieran aportar la harina de trigo y la zanahoria, ya que la harina de trigo utilizada es enriquecida con minerales debido a que la molienda del trigo fractura muchas células del endospermo poniendo a descubierto su contenido junto a los demás ingredientes (huevo, leche, etc.) que constituyen las panquecas, siendo la leche la que aporta la mayor cantidad. El contenido de cenizas aumenta a medida que se añade zanahoria a los tratamientos desde 1,32 hasta $2,01 \%$, por lo cual este incremento podría atribuirse al aporte mineral de este vegetal (0,8 gr/100; INN, 2002).

En la Tabla 2 se refleja un incremento de los carbohidratos, lo cual podría atribuirse a la disminución del contenido porcentual de humedad y de proteína de las panquecas; dichos valores se obtuvieron por diferencia y se cuantificaron en porcentaje, además son reportados en base húmeda, lo cual indica que a medida que se añade zanahoria disminuye el porcentaje de humedad y por ende aumentan los demás componentes.

\section{CONTENIDO DE $\beta$-CAROTENO Y MINERALES (CA, FE Y P):}

El contenido de $\beta$-caroteno de la mezcla de los ingredientes crudos y de las panquecas cocidas en los diferentes tratamientos se presenta en la Tabla 3. Los resultados obtenidos a través del ANAVA y la aplicación de la prueba de rangos múltiples de Duncan indican diferencias entre los promedios de los tratamientos $(P<0,01)$ para

Tabla 3

Contenido de $\beta$-caroteno en muestras de mezcla de los ingredientes crudos en las panquecas cocidas y contenido de minerales ( $\mathrm{Ca}, \mathrm{Fe}$ y $\mathrm{P})$ en las panquecas elaboradas bajo diferentes tratamientos ${ }^{1 /}$

\begin{tabular}{|c|c|c|c|c|c|}
\hline Tratamientos & $\begin{array}{c}\text { Mezcla cruda } \\
\text { Contenido de } \\
\beta-\text {-caroteno } \\
(\mu \mathbf{g} / \mathbf{1 0 0} \mathbf{g} \mathbf{M})\end{array}$ & $\begin{array}{c}\text { Panqueca cocida } \\
\text { Contenido de } \\
\beta-\text {-caroteno } \\
(\mu \mathbf{g} / \mathbf{1 0 0} \mathbf{g} \mathbf{~ M})\end{array}$ & $\begin{array}{c}\mathbf{C a} \\
(\mathbf{m g} / \mathbf{1 0 0} \mathbf{g} \mathbf{M})\end{array}$ & $\begin{array}{c}\mathbf{F e} \\
(\mathbf{m g} / \mathbf{1 0 0} \mathbf{g} \mathbf{~ M}) .\end{array}$ & $\begin{array}{c}\mathbf{P} \\
(\mathbf{m g} / \mathbf{1 0 0} \mathbf{g} \mathbf{M})\end{array}$ \\
\hline $\begin{array}{c}100 \% \mathrm{HT} ; \\
0 \% \mathrm{Z}\end{array}$ & - & - & $337,25^{\mathrm{a}} \pm 0,49$ & $17,03^{\mathrm{a}} \pm 0,05$ & $663,03^{\mathrm{a}} \pm 0,25$ \\
\hline $\begin{array}{c}75 \% \mathrm{HT} ; \\
25 \% \mathrm{Z}\end{array}$ & $401,44^{\mathrm{a}} \pm 0,51$ & $337,46^{\mathrm{a}} \pm 0,46$ & $384,75^{\mathrm{a}} \pm 0,48$ & $9,10^{\mathrm{b}} \pm 0,04$ & $639,12^{\mathrm{a}} \pm 0,48$ \\
\hline $\begin{array}{c}50 \% \mathrm{HT} ; \\
50 \% \mathrm{Z}\end{array}$ & $759,39^{\mathrm{b}} \pm 11,83$ & $637,89^{\mathrm{b}} \pm 9,94$ & $391,75^{\mathrm{a}} \pm 0,48$ & $7,10^{\mathrm{b}} \pm 0,04$ & $600,32^{\mathrm{b}} \pm 0,29$ \\
\hline $\begin{array}{c}25 \% \mathrm{HT} ; \\
75 \% \mathrm{Z}\end{array}$ & $880,34^{\mathrm{b}} \pm 2,19$ & $741,23^{\mathrm{b}} \pm 1,22$ & $403,75^{\mathrm{b}} \pm 0,25$ & $5,53^{\mathrm{b}} \pm 0,05$ & $550,37^{\mathrm{b}} \pm 0,25$ \\
\hline
\end{tabular}

\footnotetext{
1/Valores promedio de cuatro repeticiones para el contenido de $\beta$-caroteno y triplicado para el contenido de minerales (Ca, Fe y P) \pm desviación estándar.

a, b, c Valores con diferentes letras en la misma columna difieren estadísticamente a una $P<0,05$.
} 
el contenido de $\beta$-caroteno, $75 \% \mathrm{HT} ; 25 \% \mathrm{Z}$ con respecto a los tratamientos $50 \% \mathrm{HT} ; 50 \% \mathrm{Z}$ y $25 \% \mathrm{HT} ; 75 \% \mathrm{Z}$.

Para el tratamiento $100 \% \mathrm{HT}$; $0 \% \mathrm{Z}$ no se detectaron cantidades experimentalmente medibles de $\beta$-caroteno, en las condiciones del ensayo (Cromatografía HPLC), estos resultados son concordantes con lo tabulado por el Instituto Nacional de Nutrición para panquecas elaboradas con harina de trigo como ingrediente principal (INN, 2002). No obstante, en los restantes tratamientos, tanto para la mezcla de los ingredientes crudos como para las panquecas cocidas, se cuantificaron cantidades significativas de $\beta$-caroteno, cuyo contenido se incrementó con el aumento de la cantidad de zanahoria (3.902,74 $\mu \mathrm{g} \beta$-caroteno/100 g Z) en los tratamientos.

Si se comparan los contenidos de $\beta$-caroteno en la mezcla cruda de ingredientes, en cada tratamiento, con los de las panquecas cocidas correspondientes se observa una reducción en los contenidos de $\beta$-caroteno en cada caso. De esos resultados se infiere que el contenido de $\beta$-caroteno en cada tratamiento se redujo aproximadamente en un $16 \%$ por efectos del tratamiento térmico, probablemente debido a isomerización de dobles enlaces, no detectándose por el cambio de configuración con respecto al estándar usado en la cuantificación (trans $\beta$-caroteno).

Baudi (1993) señaló que el procesamiento térmico de los vegetales isomeriza del 16 al 35\% del $\beta$-caroteno, transformando el isómero trans en cis, con lo cual se reduce la disponibilidad como precursor de la vitamina $\mathrm{A}$, ya que el isómero cis no es biológicamente activo. Aunque en el cocimiento de las panquecas se manejaron temperaturas de $70^{\circ} \mathrm{C}$, la reducción en el contenido de $\beta$-caroteno observada en todos los tratamientos, respecto a la mezcla de ingredientes crudos, puede ser atribuida al efecto del tratamiento térmico.

\section{EVALUACIÓN SENSORIAL}

La prueba de Kruskall-Wallis arrojó diferencias altamente significativas para los tratamientos con respecto a las variables estudiadas (sabor, olor, color y textura), siendo las proporciones de harina de trigo y zanahoria las que hacen esta marcada diferencia entre los tratamientos (Tabla 4). El tratamiento con 50/ HT y 50\% Z resultó el de mayor aceptación.

\section{CONCLUSIONES}

La adición de zanahoria a las panquecas elaboradas con harina de trigo $(50 \% \mathrm{HT} ; 50 \% \mathrm{Z}$ y $25 \% \mathrm{HT} ; 75 \% \mathrm{Z}$ ) produce un incremento de los niveles de fibra, cenizas (minerales), grasa, carbohidratos y $\beta$-caroteno.

La incorporación de zanahoria en las panquecas de harina de trigo (25\% HT; $75 \%$ Z) incrementa significativamente los niveles de calcio, pero reduce los de hierro y fósforo.

Las panquecas elaboradas con $50 \%$ de zanahoria y $50 \%$ de harina de trigo presentan una alta aceptación en cuanto a los atributos sensoriales: sabor, color, olor y textura.

La incorporación de la zanahoria en las panquecas es una condición de alta factibilidad para mejorar el valor nutritivo del producto final.

Tabla 4

Valores promedio de las variables sensoriales evaluadas para las panquecas elaboradas

\begin{tabular}{|c|c|c|c|c|}
\hline Tratamientos & Sabor & Olor & Color & Textura \\
\hline $100 \% \mathrm{HT} ; 0 \% \mathrm{Z}$ & $3,550^{\mathrm{c}}$ & $5,450^{\mathrm{b}}$ & $3,600^{\mathrm{c}}$ & $4,150^{\mathrm{b}}$ \\
\hline $75 \% \mathrm{HT} ; 25 \% \mathrm{Z}$ & $5,650^{\mathrm{b}}$ & $7,350^{\mathrm{a}}$ & $6,700^{\mathrm{a}}$ & $6,100^{\mathrm{a}}$ \\
\hline $50 \% \mathrm{HT} ; 50 \% \mathrm{Z}$ & $7,150^{\mathrm{a}}$ & $7,400^{\mathrm{a}}$ & $7,250^{\mathrm{a}}$ & $7,200^{\mathrm{a}}$ \\
\hline $25 \% \mathrm{HT} ; 75 \% \mathrm{Z}$ & $5,050^{\mathrm{b}}$ & $4,900^{\mathrm{b}}$ & $5,050^{\mathrm{b}}$ & $4,250^{\mathrm{b}}$ \\
\hline
\end{tabular}

a, b, c Valores con diferentes letras en la misma columna difieren estadísticamente a una $P<0,05$. 


\section{LITERATURA CITADA}

AOAC. Association of Official Analytical Chemists. 1980. Official Methods of Analysis. 13 th ed. Sec. 43.008-43.013. pp 736-738.

BAUDI, S. 1993. Química de los Alimentos. 3 ed . Alambra Mexicana. México. 1124 p.

BELITZ, W.; GROSCH. 1997. Química de los Alimentos. $2^{\text {da }}$ ed. Acribia. Zaragoza-España. $1087 \mathrm{p}$

BUSHWAY, R. 1986. Determination of $\alpha$-carotene and $\beta$-carotene in Some Raw Fruits and Vegetables by HihPerformance Liquid Chromatography. J Agric. Food Chem. 34:409-412.

CHARLEY, H. 2001. Tecnología de los Alimentos. Limusa. México. $767 \mathrm{p}$.

CHACÍN, F. 2000. Diseño y Análisis de Experimentos. Ediciones del Vicerrectorado Académico de la Universidad Central de Venezuela. 383 p.

CHEFTEL, J.; H. CHEFTEL. 1992. Introducción a la Bioquímica y Tecnología de Alimentos. $2^{\text {da }}$ ed. Acribia. Zaragoza- España. Vol. I. 404 p.

COVENIN. Comisión Venezolana de Normas Industriales. 1980. Alimentos: Determinación de Humedad. Norma Venezolana 1553-80. $1^{a}$ Revisión. Caracas- Venezuela. $8 \mathrm{p}$.

COVENIN. Comisión Venezolana de Normas Industriales. 1981. Alimentos: Determinación de Cenizas. Norma Venezolana 1783-81. $1^{a}$ Revisión. Caracas- Venezuela. $6 \mathrm{p}$.
COVENIN. Comisión Venezolana de Normas Industriales. 1981. Alimentos: Determinación de Fibra Cruda. Norma Venezolana 1789-81. $1^{\text {a }}$ Revisión. Caracas-Venezuela. $6 \mathrm{p}$.

COVENIN. Comisión Venezolana de Normas Industriales. 1981. Alimentos: Determinación de Grasa. Norma Venezolana 1785-81. $1^{a}$ Revisión. Caracas- Venezuela. $7 \mathrm{p}$.

COVENIN. Comisión Venezolana de Normas Industriales. 1995. Alimentos: Determinación de Proteínas. Norma Venezolana 1195-95. $1^{\mathrm{a}}$ Revisión. Caracas- Venezuela. $10 \mathrm{p}$.

INN. Instituto Nacional de Nutrición. 2002. Tabla de Composición de Alimentos. Legumbres. Caracas-Venezuela.

MAHECHA LA TORRE, G. 1985. Evaluación Sensorial y Control de la Calidad de los Alimentos. México.

MALDONADO, R.; PACHECO, E. 1998. Elaboración de Pasta Alimenticia por Sustitución de Harina de Trigo y Zanahoria. Revista Facultad de Agronomía. UCV 24: 89104. Venezuela. Disponible: http://www.redpav_fpolar.info. ve/fagro.v24_2/m242a002.html [Consulta: 23/10/02].

MÉNDEZ, A. 2001. Contenido de Vitamina A en Alimentos Vegetales de Mayor Consumo [Documento en línea]. Disponible: http://www.sightandlife.org/sightandlife/info/ manualspan/sp02.pdf. [Consulta: 21/06/02]

SAS. (Statistical Analysis System). 1998. User's guide Statistics. Ver. 6.08. Sas. Int. Inc, Cary, N.C 